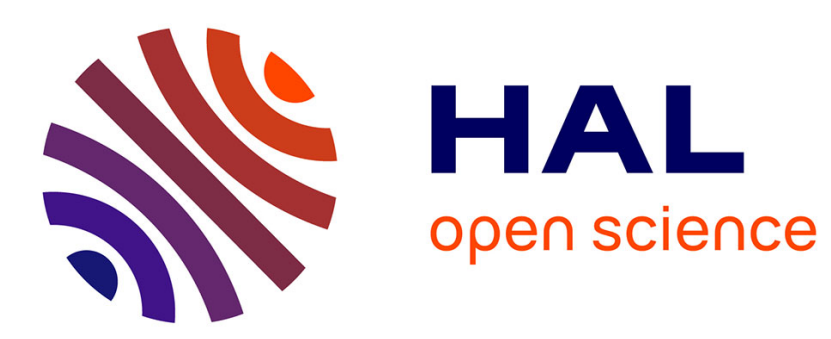

\title{
Forêts et finances communales dans le Doubs : incidence des revenus monétaires bruts des forêts communales sur le budget des collectivités locales
}

\author{
D. Mathieu, A. Robert
}

\section{- To cite this version:}

D. Mathieu, A. Robert. Forêts et finances communales dans le Doubs: incidence des revenus monétaires bruts des forêts communales sur le budget des collectivités locales. Revue forestière française, 1985, 37 (5), pp.405-412. 10.4267/2042/21828 . hal-03423916

\section{HAL Id: hal-03423916 https://hal.science/hal-03423916}

Submitted on 10 Nov 2021

HAL is a multi-disciplinary open access archive for the deposit and dissemination of scientific research documents, whether they are published or not. The documents may come from teaching and research institutions in France or abroad, or from public or private research centers.
L'archive ouverte pluridisciplinaire HAL, est destinée au dépôt et à la diffusion de documents scientifiques de niveau recherche, publiés ou non, émanant des établissements d'enseignement et de recherche français ou étrangers, des laboratoires publics ou privés. 


\title{
économie et forêt
}

\section{FORÊTS ET FINANCES COMMUNALES DANS LE DOUBS: INCIDENCE DES REVENUS MONÉTAIRES BRUTS DES FORÊTS COMMUNALES SUR LE BUDGET DES COLLECTIVITÉS LOCALES ${ }^{(1)}$}

\author{
D. MATHIEU - A. ROBERT
}

LIMINAIRE

L'excellent article de Messieurs Mathieu et Robert repose sur une analyse des donnees relatives aux années 1978 à 1980 .

Toutefois, le lecteur de 1985 doit savoir que la conjoncture s'est sensiblement dégradée au détriment de la forêt depuis cette époque. En comparant la moyenne des années 1978, 1979 et 1980 à l'année 1984, on peut voir, en effet, que dans le Doubs, les cours des grumes feuillues ne se sont pas accrus de plus de $10 \%$ alors que les bois d'cuure résineux perdaient $5 \%$ de leur valeur en francs courants et que le coût de la vie, de son côté, augmentait d'environ $70 \%$. Dans ce contexte général défavorable au revenu forestier, l'avantage relatif dont bénéficiaient naguère les forêts résineuses, s'est bien émoussé en cinq ans.

D'autre part, si, comme le soulignent à juste titre les auteurs, il existe une partition régionale assise en grande partie sur l'étagement de la végétation, on peut aussi citer deux autres facteurs qui contribuent à creuser l'écart:

- l'intérêt longtemps accordé à la production de bois de feu, au détriment du bois d'œuvre, dans les forêts feuillues, et la gestion extensive qui en découle: persistance du taillissous-futaie ou amorce, encore trop récente, de la conversion;

(1) Cet article est paru sous une forme plus étoffée dans les "Cahiers de Géographie de Besançon. Séminaires et Notes de Recherches ", $n^{\circ} 26,1983$. 


\section{MATHIEU - A. ROBERT}

- l'importance dans les mêmes forêts, des volumes de bois qui, distribués en nature sous forme d'affouage, engendrent peu de recettes financières apparentes.

En raison des difficultés actuelles du marché des résineux, les communes du "haut pays" n'ont-elles pas l'obligation de ré-employer dans leurs forêts une proportion accrue de leurs recettes domaniales, si elles veulent maintenir la qualité de leur capital producteur?

Les autres, beaucoup plus nombreuses même dans le Doubs, ne doivent-elles pas chercher au plus vite les moyens qui leur permettront demain d'avoir des peuplements plus rémunérateurs?

Telles sont les véritables questions que pose l'intéressante étude qui suit.

\section{GUIBERT}

Directeur régional de I'OFFICE NATIONAL DES FORÉTS 14, rue Plançon B.P. 329 - 25017 BESANÇON CEDEX

D'après l'Inventaire communal de 1979-80, les communes du Doubs sont propriétaires de la moitié (117000 hectares) des forêts du département. Sur les 586 communes, si seules 5 n'ont aucune propriété forestière, 530 ont au moins 50 hectares, et même près d'une commune sur 5 possède plus de 300 hectares boisés.

Dans toutes les petites régions, les mêmes catégories se retrouvent dans des proportions à peu près semblables, avec cependant une représentation légèrement plus forte des catégories supérieures à 200 hectares dans le haut-pays (dans l'arrondissement de Pontarlier, près du tiers des communes possèdent chacune plus de 300 hectares de forêts, alors que très rares sont celles qui ont moins de 50 hectares). La surface forestière moyenne possédée par chaque commune du haut-pays est de 224 hectares, contre 189 hectares dans le bas-pays.

Autrefois surexploitées et de piètre valeur, les forêts communales ont acquis depuis un siècle et demi, souvent sous la gestion des services des Eaux et Forêts, une valeur et un rendement importants. Dans les communes rurales, les ventes de bois alimentent une part notable, mais très variable dans l'espace, des budgets locaux.

Les bases de la présente étude - les comptes financiers des communes du Doubs de trois années successives 1978-1979-1980 - ne peuvent fournir qu'un aspect, certes primordial, des contributions de la forêt aux finances communales. Nous n'abordons pas ici la rentabilité financière nette des propriétés, c'est-à-dire la balance entre les dépenses occasionnées entretien, investissements de plantation et d'aménagement... - et les apports très divers qu'elles engendrent, directement par les recettes des ventes de bois d'œuvre et d'affouage, indirectement en suscitant ou en maintenant des activités locales qui ont elles-mêmes, par leurs impôts et taxes professionnelles, un impact sur le budget (emplois forestiers, scieries, industries du bois, voire activités touristiques). II n'est pas non plus dans notre propos de faire l'analyse des ressources procurées aux familles par les distributions d'affouage, petits bois et houppiers répartis gratuitement ou sous faible dépense, entre les membres de la collectivité communale. II convient cependant de considérer que dans certaines forêts de plaine, peuplées de feuillus, une part notable du revenu produit par les propriétés communales échappe au budget local et donc à notre évaluation.

Parmi les produits domaniaux forestiers comptabilisés, le revenu des coupes effectuées régulièrement selon le plan de gestion établi par l'Office national des Forêts constitue la plus grande part, de très loin. Parfois, peuvent apparaître de "menus produits forestiers", perception de certains droits et redevances souvent liées à l'affouage, ne dépassant cependant jamais plus de $5 \%$ des revenus fournis par les coupes, même en plaine. 


\section{L'APPORT FINANCIER DES COUPES FORESTIĖRES}

Pour les trois années financières étudiées $(1978,1979,1980)$, et pour l'ensemble du Doubs - à l'exclusion des 6 communes de plus de 10000 habitants en 1975 pour lesquelles le compte "produit domaniaux" n'est pas détaillé -, les coupes de bois ont rapporté $287782000 \mathrm{~F}$, soit en moyenne 96 millions de francs par an. Cette somme représente environ $80 \%$ de la totalité des revenus domaniaux des communes.

Tableau I: $\quad$ Revenus des coupes de bois selon les régions

\begin{tabular}{|c|c|c|}
\hline & $\begin{array}{l}\text { Revenus des coupes } \\
\text { de bois (en } 1000 \mathrm{~F})\end{array}$ & $\begin{array}{l}\text { Part dans le } \\
\text { total Doubs (1) }\end{array}$ \\
\hline Région bisontine ${ }^{(1)}$ & 28518,2 & $9,9 \%$ \\
\hline Vallée moyenne .. & 23714,8 & $8,2 \%$ \\
\hline Pays de Montbéliard ${ }^{(1)} \ldots \ldots$ & 10978,9 & $3,8 \%$ \\
\hline Plateaux moyens $\ldots \ldots \ldots$. & 113962,1 & $39,6 \%$ \\
\hline Haut-Doubs ${ }^{(1)} \ldots \ldots \ldots \ldots$. & 110607,9 & $38,4 \%$ \\
\hline
\end{tabular}

(1) Hors villes de plus de 10000 hab. en 1975 - pour les trois annèes 1978-79 et 80.

Dans le bas-pays, de Besançon à Montbéliard, les coupes forestières ont rapporté à l'ensemble des communes de moins de 10000 habitants, quelque 21 millions de francs par an, alors que la moitié haute du département a encaissé près de quatre fois cette somme.

Une simple carte (figure 1) révèle nettement deux zones très contrastées. La limite (indiquée en grisé sur cette carte cf. page 408), de Saint-Hippolyte à Vercel et Amancey, se moule assez rigoureusement sur l'altitude 700-800 m, niveau séparant deux grands étages de végétation: montagnard inférieur peuplé de feuillus au Nord-ouest, montagnard supérieur avec conifères au Sud-est.

Dans le bas-pays, la moitié des communes perçoit moins de $11500 \mathrm{~F}$ par an, et seules 24 (6\% des communes du secteur) ont un revenu forestier moyen qui dépasse $70000 \mathrm{~F}$. Sur les plateaux moyens et dans le Haut-Doubs, les revenus forestiers sont supérieurs à $70000 \mathrm{~F}$ pour les deux tiers des communes, et pour 28 d'entre elles le rapport atteint $350000 \mathrm{~F}$ et plus. Les chiffres mettent en évidence la richesse brute que certaines communes tirent de leur forêt, notamment dans le haut-pays qui s'adjuge quelque $78 \%$ des revenus bruts des coupes annuelles, bien que les propriétés n'y soient guère plus importantes que dans le reste du département.

Le rapport entre les revenus bruts des coupes de bois et la surface des forêts communales définit le rendement financier brut à l'hectare. Les variations sont considérables, de quelques dizaines de francs à plus de $5000 \mathrm{~F}$. Elles mettent en évidence les différences liées à la nature des peuplements (feuillus et conifères), à leur mode de gestion (futaie ou taillis) et à la destination de leurs produits (dont une part en plaine échappe par l'affouage à la prise en compte dans les recettes communales).

Dans le haut-pays, les meilleurs rapports sont obtenus dans les cantons les plus septentrionaux: Maîche, Le Russey, Pierrefontaine, Morteau, Montbenoît et Levier, avec une moyenne de $2300 \mathrm{~F}$ par hectare et par an. Dans cet ensemble, trois secteurs apparaissent très favorisés: le plateau des Franches-Montagnes au nord (cantons de Maîche et Le Russey), le Val de Vennes et le plateau d'Avoudrey, le Val de Morteau et le Saugeais, où le revenu annuel moyen dépasse $3000 \mathrm{~F}$ par hectare. C'est d'ailleurs dans ces secteurs que s'est produite, au cours des dernières décennies, une forte extension des boisements privés et publics: l'excellent rapport des forêts, et singulièrement des forêts communales, a certainement été la meilleure des incitations aux plantations de conitères, principalement d'épicéas. 


\section{MATHIEU - A. ROBERT}

Au sud de Pontarlier, la situation est moins favorable, le revenu légèrement plus faible. La croissance plus lente des arbres, du fait d'une rigueur climatique accentuée par l'altitude, le moins bon entretien des boisements gêné par la vigueur des pentes et les difficultés d'accès, la présence de prés-bois encore exploités par l'élevage, expliquent au moins pour partie ces résultats plus médiocres.

Cependant la véritable frontière est à l'altitude de $750 \mathrm{~m}$ qui coupe en deux les cantons d'Amancey, de Vercel et Pierrefontaine. Le rapport des forêts communales y chute de façon brutale, et systematique en dessous de $1000 \mathrm{~F}$ par hectare et par an. Les valeurs les plus faibles se rencontrent dans l'ouest du département (cantons d'Audeux, Boussières, Quingey) où de médiocres taillis procurent aux communes des fonds dérisoires.

Rapporté à la population résidente (figure 2), l'apport financier des coupes de bois dans les budgets des communes de moins de 10000 habitants s'élève à $364 \mathrm{~F}$ par habitant et par an (2).

(2) A titre de comparaison et en prenant cette fois-ci l'ensemble des produits domaniaux, le revenu moyen pour ces mêmes communes est de $427 \mathrm{~F}$ (la forêt représente donc $85 \%$ du total) mais seulement de $47 \mathrm{~F}$ pour les communes de plus de 10 o00 habitants: c'est dire que dans ces dernières, le rôle des revenus forestiers est trés marginal.

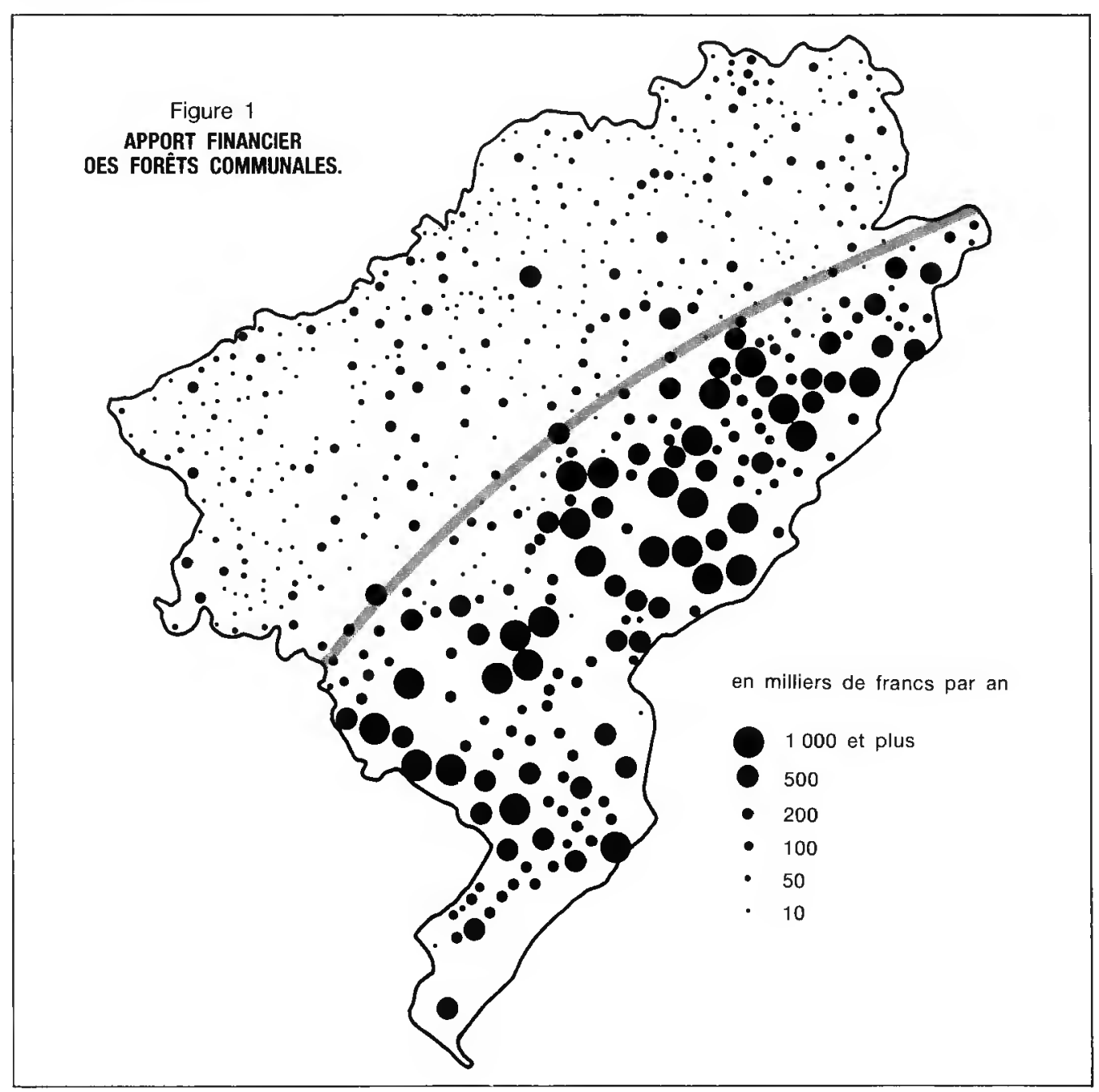




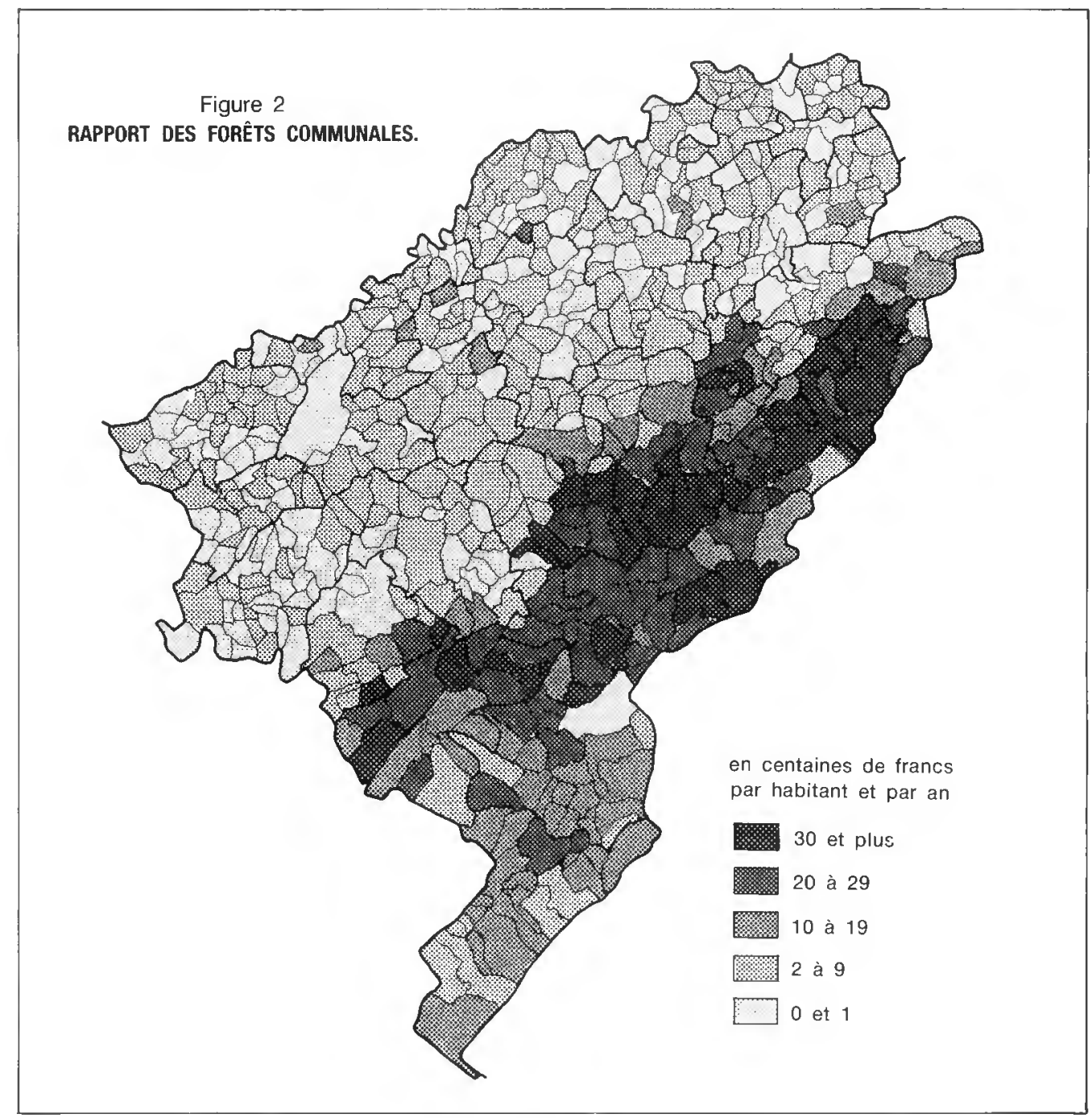

L'opposition entre haut et bas-pays persiste: les plus hauts revenus per capita (supérieurs à $1000 \mathrm{~F})$ sont pratiquement tous situés au sud de la ligne Saint-Hippolyte-Vercel-Amancey. Mais la netteté de la division s'estompe, et à l'intérieur des deux ensembles, apparaissent d'importants contrastes qui traduisent surtout les différences de poids démographique des communes. Dans le bas-pays, les régions urbaines s'individualisent nettement, avec des revenus forestiers inférieurs à $200 \mathrm{~F}$ par habitant : tout le pays de Montbéliard, la banlieue bisontine, mais aussi les agglomèrations et cités industrielles de la moyenne vallée du Doubs (Baume-les-Dames, Clerval, I'Isle-sur-le-Doubs...).

Dans le haut-pays, les écarts sont d'autant plus accusés que certaines communes richement dotées en revenus forestiers sont peu peuplées. Le revenu y dépasse parfois $3000 \mathrm{~F}$ par an et par habitant, alors que, dans les villes et bourgs, il tombe nettement en dessous de $200 \mathrm{~F}$. Plus que dans le bas-pays apparaît ici un contraste urbain-rural important. 


\section{LES REVENUS FORESTIERS DANS LES BUDGETS COMMUNAUX}

Pour l'ensemble du département du Doubs, les budgets communaux (moyenne des 3 années 1978, 1979 et 1980) se sont élevés à 1,7 milliard de francs, soit $3500 \mathrm{~F}$ par habitant. Cette moyenne masque de profondes disparités entre communes, puisque le minimum est de $800 \mathrm{~F}$, alors que le maximum avoisine $11000 \mathrm{~F}$.

Parmi les localités de plus de 10000 habitants, la séparation est très marquée entre les cités ouvrières de la Porte de Bourgogne - Valentigney, Audincourt, Bethoncourt - à faible charge budgétaire par habitant, et les villes-centres à vocation tertiaire affirmée - telles Pontarlier, Montbéliard et surtout Besançon - où la charge budgétaire par habitant atteint ou dépasse largement la moyenne départementale (5800 F à Besançon): les services urbains justifient des besoins financiers supérieurs.

Les autres communes semblent obéir à une triple partition. La place plus élevée de certaines communes dans la hiérarchie urbaine, et donc le niveau de services qu'elles fournissent à la population environnante, se traduisent par un accroissement des charges:

- L'opposition entre haut et bas-pays se retrouve, le premier plus riche mais aussi aux coûts de fonctionnement et d'investissement plus élevés en fonction du relief, du climat (rieige), de la dispersion de l'habitat parfois...

- Des économies d'échelle différencient les cantons proprement ruraux peu peuplés, notamment du bas-pays, des secteurs plus urbanisés ou en développement démographique rapide (banlieue bisontine).

Globalement, sur l'ensemble des communes du département, les finances représentées dans les budgets des collectivités locales ressortissent pour $7 \%$ des produits domaniaux, $15 \%$ des contributions directes, $78 \%$ de la dotation budgétaire de fonctionnement et autres ressources annexes. Dans le premier chapitre, la forêt fournit environ $85 \%$ des produits domaniaux, donc $6 \%$ de la masse budgétaire globale. Mais cette part varie selon les communes (figure 3). Elle est négligeable (moins de $1 \%$ ) dans les villes de plus de 10000 habitants.

Dans le haut-pays, les coupes de bois ont globalement fourni $22 \%$ des ressources budgétaires. Cette part dépasse $30 \%$ dans les deux tiers des communes situées au-dessus de $750 \mathrm{~m}$ d'altitude. Elle s'abaisse entre 20 et $30 \%$ dans les centres de service, petites villes et bourgs ruraux tels Pierrefontaine, Le Russey, Frasne, descend même en dessous de $20 \%$ dans les villes et cités horlogères: Villers-le-Lac et Morteau, Charquemont et Damprichard, Maîche.

Dans le bas-pays, non comprises les villes de plus de 10000 habitants, l'apport brut de la forêt s'établit à $7 \%$ du revenu budgétaire. Mais, alors que dans la région bisontine et le pays de Montbéliard il ne dépasse guère $5 \%$, dans les cantons ruraux de la moyenne vallée du Doubs, comme dans celui de Quingey plus au sud, il oscille entre 10 et $20 \%$, avec toutefois, et fort logiquement, une part plus réduite dans les bourgs et les petits centres urbains: Baume-lesDames, Rougemont ou l'Isle-sur-le-Doubs.

Dans le même temps, les contributions directes locales ont collecté dans l'ensemble du département une somme moyenne de 335 millions de francs par an (dont il est vrai 140 millions pour la seule ville de Besançon et 51 millions pour Montbéliard). L'apport global des revenus forestiers équivaut donc à $35 \%$ du total des impôts locaux recueillis. Mais les ressources brutes tirées de la forêt sont très minimes comparées aux contributions directes, dans les villes de plus de 10000 habitants (moins de $4 \%$ sauf à Pontarlier) comme dans tout le pays de Montbéliard; alors que les rapports sont à peu près équivalents dans la périphérie bisontine et avec un certain avantage pour les revenus forestiers dans les cantons plus ruraux du bas-pays. Enfin dans la moitié sud-est du département, les 12 millions de francs que représentent les impositions locales comptent peu à côté des quelque 75 millions de francs tirés chaque année des 
Figure 3

PART DES RESSOURCES FORESTIĖRES DANS LE BUDGET GLOBAL DES COMMUNES.

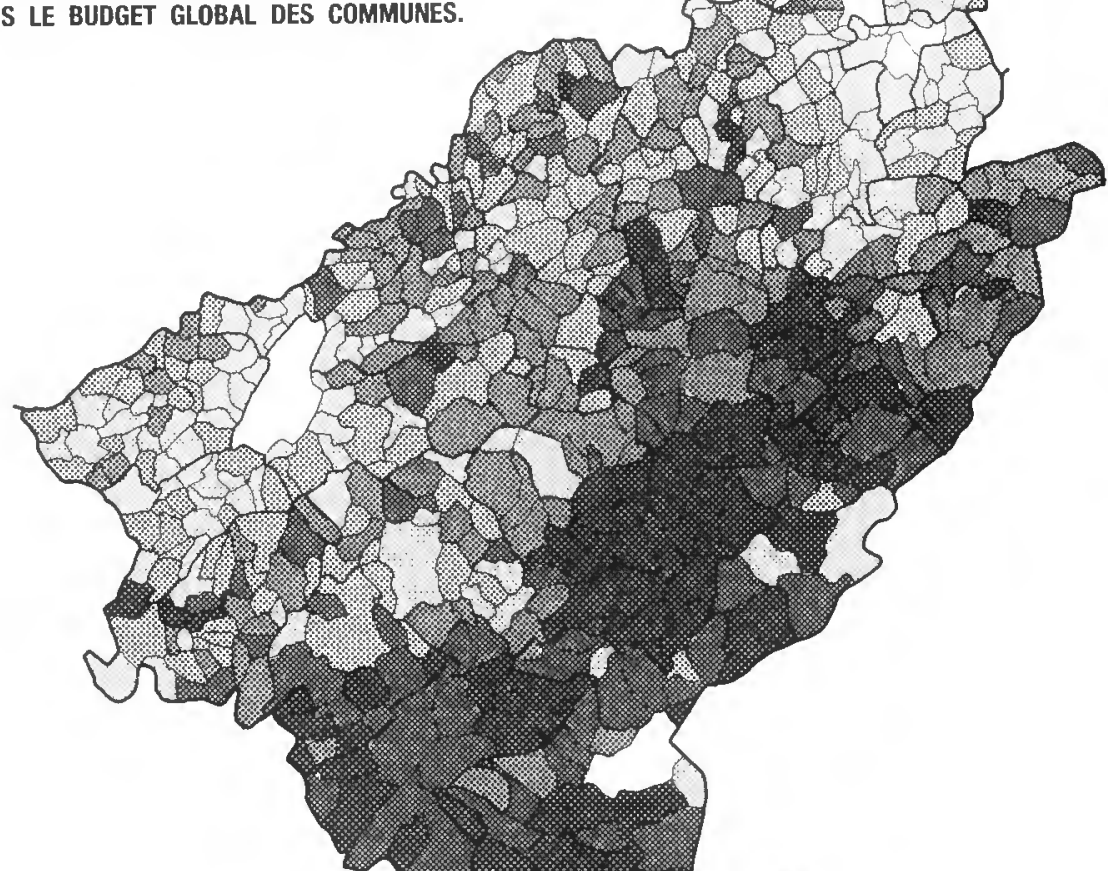

$30 \%$ et pius

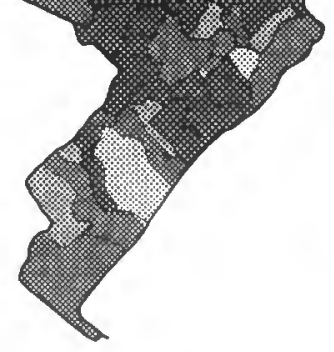

20 à $29 \%$

10 à $19 \%$

\% 5 à $9 \%$

$\square$ moins de $5 \%$

Communes de plus de 10000 habitants en 1975

coupes de bois. Dans les secteurs les plus riches, l'apport financier que représentent ces dernières évite même parfois toute fiscalité locale, (haut-Dessoubre, Val des Usiers, bordure du dèpartement d'Arc-sous-Montenot à Gellin) et dans certaines communes, les municipalités ont tenté de mettre en pratique un "impôt négatif " en faisant bénéficier directement les familles de la « manne " forestière.

\section{CONCLUSIONS}

Plus que la superficie des propriétés forestières, ce sont les rendements financiers liès aux essences dominantes qui introduisent les disparités majeures dans les ressources budgétaires propres des communes, et qui modifient largement la répartition de la fiscalité locale et les 


\section{MATHIEU - A. ROBERT}

choix budgétaires des municipalités. Globalement deux grandes partitions peuvent être décelées:

- Une partition régionale, assise en grande partie sur l'étagement de la végétation et les aptitudes des boisements, privilégiant une bande large d'une vingtaine de kilomètres sur les plateaux moyens au-dessus de $750 \mathrm{~m}$ d'altitude, de Maîche à Levier, et Amancey. Chaque municipalité peut y compter sur un revenu forestier annuel important, de plusieurs centaines de milliers de francs, quelquefois plus d'un million, avec un fort rendement financier à l'hectare. Lorsque la population est faible, il est possible de réduire, voire d'ignorer les impôts locaux. Dans la Haute-Chaîne, à des altitudes plus fortes, dans un paysage plus heurté et marqué par un climat plus rigoureux, les rendements forestiers, quoique légèrement moindres, laissent généralement aux budgets municipaux des fonds suffisants pour leur permettre une faible pression fiscale.

Le bas-pays est beaucoup moins favorisé. Si la région autour de Baume-les-Dames conserve encore quelques traits de la situation précédente (impôts locaux réduits dans nombre de communes), les produits de la forêt sont nettement plus faibles, tant par rapport à la superficie boisee qu'au nombre d'habitants. Une part cependant du croît de la forêt - ici de feuillus échappe au budget communal sous forme d'affouage. Région bisontine et pays de Montbéliard enfin se caractérisent par l'indigence des revenus forestiers rapportés au nombre d'habitants et à l'importance des budgets locaux qui doivent davantage compter sur les contributions directes.

- Une partition fonctionnelle, entre types de communes, séparant les communes agricoles, d'une part, des autres localités industrielles ou tertiaires souvent plus peuplées, d'autre part. Dans ces dernières, l'apport financier lié aux forêts, rapporté à la population comme à l'ensemble du budget est généralement mince. De même dans les bourgs et villes à fonctions tertiaires, la part du budget représentée par les revenus forestiers compte souvent peu vis-à-vis de la pression fiscale et son poids est inverse du rôle du centre dans la hiérarchie urbaine. Ce n'est vraiment que dans les communes rurales traditionnelles, à dominante agricole, que les forêts communales jouent un rôle majeur dans les finances locales et l'établissement du budget.

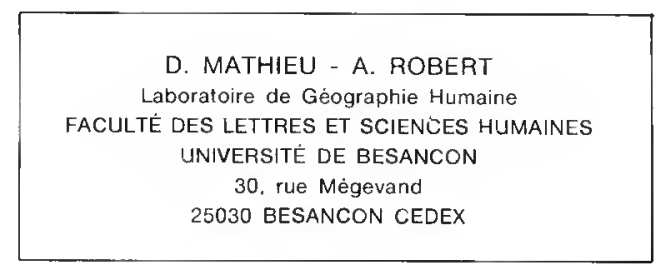

